\title{
Retrospective-comparative study of patients with obstructive sleep apneas and hypopneas in awake endoscopy and drug induced sleep endoscopy
}

\author{
Alberto Eugenio Rabino, Melissa Castillo Bustamante* and María Andrea Ricardo \\ Department of Otorhinolaryngology, Buenos Aires British Hospital, Buenos Aires, Argentina
}

\begin{abstract}
Background: This study describes endoscopic findings in patients with obstructive sleep apnea hypopnea (OSA) who were first assessed through awake endoscopy and then drug-induced sleep endoscopy (DISE).

Objective: To compare awake versus drug-induced sleep endoscopy findings to assess their relative value in final therapeutic decisions.

Materials and methods: This analytical, retrospective study included $>18$-year old OSA patients in the period between June 2013 and June 2015 . The statistical analysis was performed with PSPP and the data were interpreted with the chi-square test.

Results: The mean age was 45.88 ( \pm 1.03 ). OSA was more prevalent in men and polysomnography (PSG) revealed that $33.3 \%$ of male patients had severe OSA Friedman Stage II was the most frequent finding observed in patients evaluated with both endoscopic approaches (57.97\%). Velum, tongue, and epiglottis showed significant differences in collapse and airflow patterns. Most cases of partial and complete collapse were concentric.
\end{abstract}

Conclusion: DISE in patients with mild, moderate, and severe OSA revealed clinically significant obstructions in the velum, tongue, and epiglottis as well as partial and complete concentric collapse. These findings could be useful to guide future therapeutic decisions.

\section{Introduction}

OSA, central sleep apnea, and sleep-related hypoventilation syndromes are sleep-related breathing disorders. The estimated prevalence of these disorders is $9 \%$ for women and $24 \%$ for men between 30 and 60 years of age [1]. In the general population, OSA prevalence is $1-5 \%$ [1]. The tool used to diagnose this disorder is PSG with overnight pulse oximetry $[2,3]$. The first choice treatment for patients with moderate and severe OSA or symptomatic sleepiness is $\mathrm{CPAP}$, followed by surgery-for patients with poor adaptation to CPAP or to optimize treatment adherence [3]. The literature also mentions indication for surgery for mild OSA patients with good adaptation to CPAP [4].

Surgery candidates are screened by physical examination and supplementary procedures such as awake and drug-induced sleep endoscopy [4].

The purpose of this study is to compare awake versus drug-induced sleep endoscopy to assess their relative value in final therapeutic decisions.

\section{Materials and methods \\ Design}

This is a retrospective analytical study of 120 patients aged $>18$ years with a diagnosis of OSA and indication for surgery who received care at the Department of Otorhinolaryngology of a four-level hospital between June 2013 and June 2015.
Patients were assessed in the outpatients' service of the Department of Otorhinolaryngology. The diagnostic assessment protocol included awake endoscopy followed by DISE 20 days later.

\section{Study population}

Three hundred forty-eight patients consulted for OSA. Initial evaluation was performed by nocturnal PSG. OSA was defined as Apnea Hypopnea Index $(\mathrm{AHI})>5$.

PSG revealed that 47 patients had mild OSA (AHI between 6 and 15), 136 had moderate OSA (AHI between 16 and 30), and 149 had severe OSA $(\mathrm{AHI}>30)$.

Data were missing for 16 patients who were, therefore, excluded.

Out of these patients, 41 were diagnosed with moderate and severe OSA or OSA with daytime sleepiness (defined as Epworth Sleepiness Scale (ESS) score $\geq 12$ ) [5] and received an indication for CPAP but were referred for surgery after refusing this treatment option. Fortyseven patients diagnosed with moderate or severe OSA, with an indication for CPAP, were referred for surgery due to failure to adapt

Correspondence to: Melissa Castillo Bustamante, Department of Otorhinolaryngology, Buenos Aires British Hospital, Buenos Aires, Argentina, Tel: +54 91143096400 - 2622; E-mail: melissacastillobustamante@gmail.com

Key words: apnea, endoscopy, otorhinolaryngology, pharynx

Received: September 02, 2017; Accepted: September 22, 2017; Published: September 25, 2017 
to CPAP therapy. Three patients with moderate or severe OSA and adequate use of CPAP preferred surgery for relief of their symptoms.

Twenty-nine patients with mild OSA also received an indication for surgery. Therefore, surgical treatment was indicated to 120 study subjects (Figure 1).

PSG findings revealed 29 patients with mild OSA (24.2\%), 45 patients with moderate OSA ( $37.5 \%)$, and 46 patients with severe OSA (38.3\%) (Figure 1).

Enrolled patients signed an informed consent form with information about study duration and procedures which guaranteed data confidentiality and protection of participants' identity. Such form is attached at the end of this study.

This study was approved by the Institutional Review Board of Buenos Aires British Hospital in October 2016, which is a member of the Institutional Review Boards of the City of Buenos Aires, Argentina.

\section{Inclusion and exclusion criteria}

Inclusion criteria: patients diagnosed with OSA through PSG with overnight pulse oximetry or respiratory polygraphy with poor adaptation to CPAP therapy.

Exclusion criteria: patients aged $<18$ years, with good CPAP adaptation, high cardiovascular risk (ASA $\geq 3$ ), whose data was missing, or refused to take part in the study.

\section{Data collection}

Data were collected using a password protected database managed by researchers at the Department of Otorhinolaryngology of Buenos Aires British Hospital, Argentina. Data collected included: demographic and anthropometric characteristics as available in patients' clinical histories, age (years), gender (male/female), Body Mass Index (BMI: $\mathrm{kg} / \mathrm{m}^{2}$ ), Epworth Sleepiness Score ${ }^{5}$ (continuous numerical values ranging between 0 and 24, with a clinical threshold of >12). Craniofacial conformation (CFC) was classified into the following types: dolichocephalic, mesocephalic, and brachycephalic. Occlusion was classified into Class 1,2, or 3. Mallampati (M) scale [6]: scale 1, 2, 3, and 4. Friedman-modified Mallampati classification ${ }^{7}: 1,2 \mathrm{a}$, 2b, 3, and 4. Tonsillar size: continuous scale 0, 1, 2, 3, and 4; Friedman classification [7]: I, II, and III.

Awake endoscopy was performed in an office setting. Müller's maneuver [8] and/or simulation of snoring were used to assess patients' nose, oropharynx, and hypopharynx. DISE was performed approximately 20 days later in the operating room using a single dose of midazolam $(0.05 \mathrm{mg} / \mathrm{kg}$ or $1.5 \mathrm{mg})$, followed by propofol (1.5-3 ug/ $\mathrm{ml}$ ) via infusion pump, according to the protocol attached.

Endoscopic findings are reported according to the modified VOTE classification [9] 99V: velum; O: oropharynx; T: tongue base; and E: epiglottis. Each structure within the laryngopharynx was described separately: $V$ : nasopharynx, velum, uvula, and lateral walls; $O$ : palatine tonsils and lateral walls; $T$ : tongue base, lingual tonsils, and lateral walls; and $E$ : "a" if the supraglottis is involved and "b" if the glottis is involved. Collapse is described according to its anatomic configuration: A (anteroposterior), L (lateral), or C (concentric). Collapse is further classified into Grade 2 (complete), Grade $\mathbf{1}$ ( $>50 \%$ narrowing of upperairway lumen or incomplete collapse), or Grade $\mathbf{0}$ (only vibration without actual narrowing of pharyngeal lumen).

\section{Statistical analysis}

The results of the statistical analysis of mentioned variables are summarized in tables and charts, with measures of absolute and relative frequency; PSPP 0.10 .1 was used to measure central tendency and variability; T-test was applied to assess the association between collapse and the results for each variable of the VOTE classification. The chi square test $(\mathrm{p}<0.05)$ was used to evaluate statistical significance. Results were presented as mean and standard deviation for studied anthropometric and demographic variables.

\section{Results}

\section{General characteristics}

$82 \%$ of intervened patients were men $(n=114)$. The age of the overall study population ranged between 18 and 69 , with a mean value of $45.88( \pm 1.03)$.

On physical examination, patients' mean BMI was $30.44( \pm$ 1.65)-ranging from 20.58 to $43.09 \mathrm{~kg} / \mathrm{m}^{2}$. The mean value of ESS (daytime sleepiness) was $10.13( \pm 0.45)$. The study population presented the following craniofacial anatomy: $65.2 \%$ mesocephalic, $23.2 \%$ dolichocephalic, and $10.9 \%$ brachycephalic; $42.7 \%$ with Class-I occlusion, $41.32 \%$ with Class-II occlusion, and $16.0 \%$ with Class-III occlusion. According to Friedman's classification, the most frequent finding was stage II ( 80 patients; $57.97 \%$ ), followed by stage III (42 patients; $30.44 \%$ ), and stage I (16 patients; $11.59 \%$ ) (Table 1).

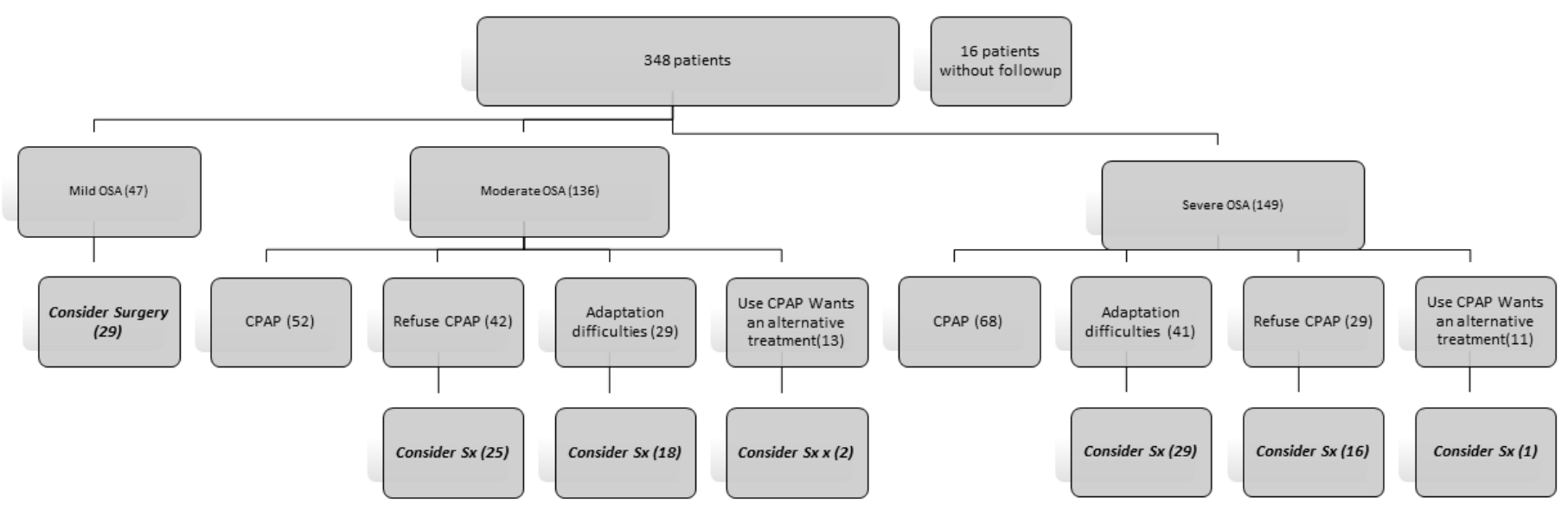

Figure 1. Subjects diagnosed with OSA. 
Table 1. Demographic characteristics and anthropometric values of study population.

\begin{tabular}{|l|l|}
\hline \multicolumn{2}{|l|}{ Demographic Characteristics of Study Population } \\
\hline $\mathrm{N}$ & 120 \\
\hline Age (years) & 45.88 years $( \pm 1.03)$. \\
\hline$\%$ Men & 82 \\
\hline Body Mass Index $\left(\mathrm{kg} / \mathrm{m}^{2}\right)$ & $30.44( \pm 1.65)$ \\
\hline Epworth daytime Sleepiness Scale & $10.13( \pm 0.45)$ \\
\hline Anthropometric Characteristics of the General Population \\
\hline Brachycephalic anatomy & $10.9 \%$ \\
\hline Mesocephalic anatomy & $62.5 \%$ \\
\hline Dolichocephalic anatomy & $23.2 \%$ \\
\hline Occlusion Pattern I & $42.7 \%$ \\
\hline Occlusion Pattern II & $41.3 \%$ \\
\hline Occlusion Pattern III & $16 \%$ \\
\hline Friedman I & $11.5 \%$ \\
\hline Friedman II & $57.9 \%$ \\
\hline Friedman III & $30.4 \%$ \\
\hline
\end{tabular}

As for the modified VOTE classification, $50 \%$ of the patients classified as Velum 0 were identified by DISE while the remaining $50 \%$ were identified by awake endoscopy. $70 \%$ of Velum 1a and $90 \%$ of Velum $1 \mathrm{c}$ cases were identified by awake endoscopy. $60 \%$ of the population classified as Velum 2a and 2c were identified by DISE (Figure 2).

In terms of oropharyngeal assessment, $60 \%$ of collapse 0 cases were identified by awake endoscopy; $50 \%$ of collapse 11 cases were identified by DISE; $100 \%$ of $1 \mathrm{c}$ and $80 \%$ of $2 \mathrm{l}$ cases were identified by awake endoscopy. While $100 \%$ of $2 c$ cases were described by DISE (Figure 2 ).

As for the tongue, $60 \%$ of 0 cases as well as $50 \%$ of $1 \mathrm{a}$ and $60 \%$ of $1 \mathrm{c}$ cases were identified by awake endoscopy. $60 \%$ of 11 and $90 \%$ of $2 \mathrm{a}$ cases, respectively, were identified by DISE. $80 \%$ of $2 c$ and $100 \%$ of 21 were identified by DISE (Figure 2).

In epiglottis findings, $60 \%$ of 0 cases were identified by awake endoscopy; and 80 and $95 \%$ of $1 \mathrm{a}$ and $2 \mathrm{a}$ cases, respectively, were identified by DISE (Figure 2).

In this comparative study of awake endoscopy versus DISE, a chi square test $(p<0.05)$ revealed statistically significant results for the following variables: Velum $1 \mathrm{c}, 2^{\mathrm{a}}$, and 2c; Oropharynx 1c; Tongue 0; and Epiglottis $1^{\mathrm{a}}$ (Table 2).

\section{Discussion}

OSA evaluation requires extensive physical examination, imaging, and procedures such as awake endoscopy and DISE [10]. According to American and European literature, DISE is mainly indicated for: 1) moderate and severe OSA patients without morbid obesity who cannot tolerate CPAP as a diagnostic test before indication for surgery or mandibular advancement device; 2) patients with mild OSA or simple snoring with an indication for surgery or mandibular advancement device; and 3) patients with OSA who do not respond to surgery [11]. DISE findings are reported using VOTE classification, which includes information about the velum, lateral walls of the oropharynx, tongue, and epiglottis [11]. This classification has been used by authors like Sher et al. [12] and Sadaoka et al. [13], who described it as easy to apply and reproduce. It also allows maneuvers such as manual closure of the mouth and the Esmarch maneuver by mandibular advancement [14].

In some studies, DISE has been reported to improve the selection of procedures and outcomes and has proven to be a reliable method to assess the airways and the hypopharynx, where awake endoscopy makes assessment more difficult. The mean age of this study is consistent with that of other studies as authors as Marais [15]. Likewise, in those studies the intervened population was mostly made up of male patients.

As for patients assessed by PSG ( $\mathrm{n}=138), 29$ patients were diagnosed with mild OSA (24.2\%), 45 with moderate OSA (37.5\%), and 46 with severe OSA (38.3\%). In other studies by Steinhart et al. [16], and Kezirian [17], the percentages reported for mild, moderate, and severe OSA were $38 \%, 28.6 \%$, and $52 \%$, respectively, which is relatively close to our own findings.

The authors of this study were not able to find an assessment of craniofacial configuration and occlusion in other studies using DISE and/or awake endoscopy.

This study revealed significant differences in the VOTE classification of DISE findings, such as Velum 1c, 2a and 2c, Oropharynx 1c, Tongue 0 , and Epiglottis $1^{\text {a }}$. Clinically and statistically significant differences were observed in complete and partial concentric collapses, especially at the level of the velum, and partial collapse at the level of the oropharynx, tongue, and epiglottis. In previous studies, Capasso et al. [18] and Yoon et al. [10] observed important findings in terms of modified anatomic structures in velum, tongue, and epiglottis, but not such significant changes in the oropharynx, which is consistent with our own findings.

The authors of this study were not able to find studies comparing awake endoscopy with DISE. However, there are multiple Asian and European studies on the use of DISE $[18,19]$, in this study, DISE is presented as a useful tool to facilitate the assessment of vibration and collapse in OSA patients. According to a literature review, this is the only study conducted in Argentina with DISE and VOTE classification to report results.

Table 2. Results of Awake Endoscopy vs Drug-Induced Sleep Endoscopy (DISE) using the parameters of the VOTE Classification.

\begin{tabular}{|c|c|c|c|}
\hline & \begin{tabular}{|c|} 
AWAKE \\
$\begin{array}{c}\text { Number of events of } \\
\text { obstruction }\end{array}$
\end{tabular} & \begin{tabular}{|c|} 
DISE \\
$\begin{array}{c}\text { Number of events of } \\
\text { obstruction }\end{array}$
\end{tabular} & $\mathbf{p}$ \\
\hline \multicolumn{4}{|c|}{ Velum } \\
\hline 0 & 5 & 5 & 0.05 \\
\hline $1 a$ & 20 & 9 & 0.07 \\
\hline $1 c$ & 27 & 3 & $<0.05$ \\
\hline $2 a$ & 25 & 44 & 0.0099 \\
\hline $2 c$ & 61 & 77 & 0.0499 \\
\hline \multicolumn{4}{|c|}{ Oropharynx } \\
\hline 0 & 101 & 64 & 0 \\
\hline 11 & 21 & 22 & 1 \\
\hline $1 \mathrm{c}$ & 8 & - & 0.002 \\
\hline 21 & 7 & 2 & 0.17 \\
\hline $2 \mathrm{c}$ & 1 & 50 & 0 \\
\hline \multicolumn{4}{|c|}{ Tongue } \\
\hline 0 & 87 & 52 & $<0.05$ \\
\hline 1a & 26 & 28 & 0.877 \\
\hline $1 \mathrm{c}$ & 14 & 7 & 0.169 \\
\hline 11 & 4 & 6 & 0.748 \\
\hline $2 a$ & 5 & 34 & 0 \\
\hline $2 \mathrm{c}$ & 2 & 8 & 0.102 \\
\hline 21 & - & 3 & 1 \\
\hline \multicolumn{4}{|c|}{ Epiglottis } \\
\hline 0 & 130 & 70 & 1 \\
\hline 1a & 6 & 19 & 0.033 \\
\hline $2 a$ & 2 & 49 & 0 \\
\hline
\end{tabular}



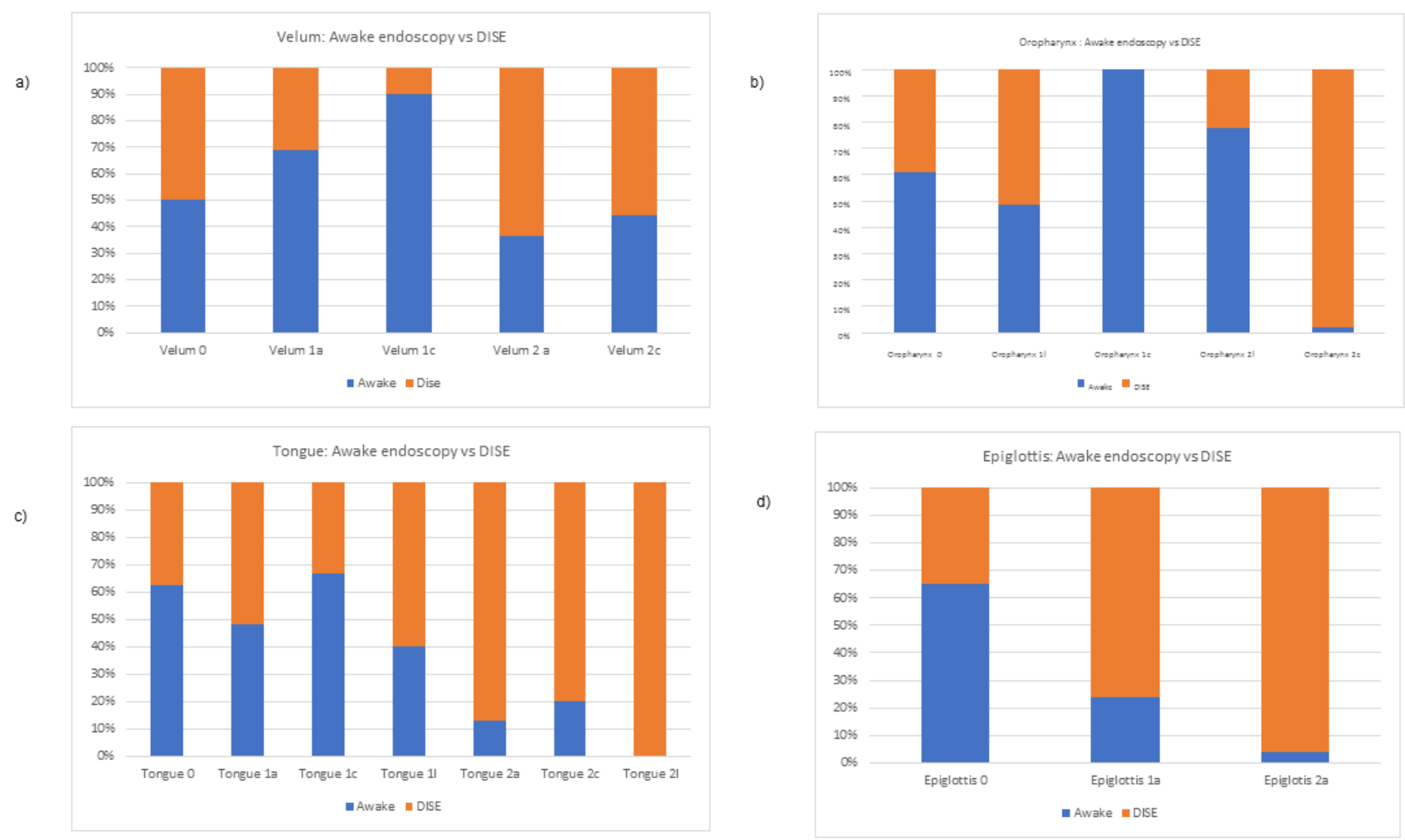

Figure 2. Results of Awake Endoscopy versus Drug-Induced Sleep Endoscopy (DISE) using the parameters of the VOTE Classification

\section{Conclusion}

This study showed that DISE allows the assessment of clinically significant obstructions at the level of the velum, tongue, and epiglottis and reveals partial and complete concentric collapse in patients with mild, moderate, and severe OSA. This information may prove useful to guide future therapeutic decisions.

\section{Disclosure}

The authors of this manuscript have no conflicts of interest.

\section{References}

1. Charakorn N, Kezirian EJ (2016) Drug-Induced Sleep Endoscopy. Otolaryngol Clin North Am 49: 1359-1372. [Crossref]

2. Carrasco Llatas M, Dalmau Galofre J, Zerpa Zerpa V, Marcano Acuña M, Mompó Romero L (2014) Drug-induced sleep videoendoscopy: clinical usefulness and literature review. Acta Otorrinolaringol Esp 65: 183-190. [Crossref]

3. Pilaete K, De Medts J, Delsupehe KG (2014) Drug-induced sleep endoscopy changes snoring management plan very significantly compared to standard clinical evaluation. $J$ Eur Arch Otorhinolaryngol 271: 1311-1319. [Crossref]

4. Ravesloot MJ, de Vries N (2011) One Hundred Consecutive Patients Undergoing Drug-Induced Sleep Endoscopy: Results and Evaluation. Laryngoscope 121: 27102716. [Crossref]

5. Doneh B (2015) Epworth Sleepiness Scale. Occup Med (Lond) 65: 508. [Crossref]

6. Mallampati SR (1983) Clinical sign to predict difficult tracheal intubation (hypothesis). Can Anaesth Soc J 30: 316-317. [Crossref]

7. Friedman M, Hamilton C, Samuelson CG, Lundgren ME, Pott T (2013) Diagnostic value of the Friedman tongue position and Mallampati classification for obstructive sleep apnea: a meta-analysis. Otolaryngol Head Neck Surg 148: 540-547. [Crossref]

8. Soares MC, Sallum AC, Gonçalves MT, Haddad FL, Gregório LC (2009) Use of Muller's maneuver in the evaluation of patients with sleep apnea--literature review. Braz J Otorhinolaryngol 75: 463-466. [Crossref]
9. Dijemeni E, D'Amone G (2017) In reference to 'Drug-induced sleep endoscopy: the VOTE classification'. Eur Arch Otorhinolaryngol. [Crossref]

10. Rodriguez-Bruno K, Goldberg AN, McCulloch CE, Kezirian EJ (2009) Test-retes reliability of drug-induced sleep endoscopy. Otolaryngol Head Neck Surg 140: 646651. [Crossref]

11. Sher AE, Schechtman KB, Piccirillo JF (1996) The efficacy of surgical modifications of the upper airway in adults with obstructive sleep apnea syndrome. Sleep 19: 156177. [Crossref]

12. Sadaoka T, Kakitsuba N, Fujiwara Y, Kanai R, Takahashi H (1996) The value of sleep nasendoscopy in the evaluation of patients with suspected sleep-related breathing disorders. Clin Otolaryngol Allied Sci 21: 485-489. [Crossref]

13. Berry S, Roblin G, Williams A, Watkins A, Whittet HB (2005) Validity of sleep nasendoscopy in the investigation of sleep related breathing disorders. Laryngoscope 115: 538-540. [Crossref]

14. Yoshida K (2000) The relationship between sleep position and therapeutic effect of the Esmarch-Scheine appliance in sleep apnea syndromes. Fortschr Neurol Psychiatr 68 : 93-96. [Crossref]

15. Marais J (1998) The value of sedation nasendoscopy: a comparison between snoring and non-snoring patients. Clin Otolaryngol Allied Sci 23: 74-76. [Crossref]

16. Steinhart H, Kuhn-Lohmann J, Gewalt K, Constantinidis J, Mertzlufft F, et al. (2000) Upper airway collapsibility in habitual snorers and sleep apneics: evaluation with druginduced sleep endoscopy. Acta Otolaryngol 120: 990-994. [Crossref]

17. Kezirian EJ (2006) Drug-induced sleep endoscopy. Op Tech Otolaryngol 17: 230-232.

18. Capasso R, Rosa T, Tsou DY, Nekhendzy V, Drover D, et al.(2016) Variable findings for drug-induced sleep endoscopy in obstructive sleep apnea with propofol versus dexmedetomidine. Otolaryngol Head Neck Surg. 154: 765-770. [Crossref]

19. Yoon BW, Hong JM, Hong SL, Koo SK, Roh HJ, et al. (2016) A comparison of dexmedetomidine versus propofol during drug-induced sleep endoscopy in sleep apnea patients. Laryngoscope 126: 763-767. [Crossref]

Copyright: (C2017 Rabino AE. This is an open-access article distributed under the terms of the Creative Commons Attribution License, which permits unrestricted use, distribution, and reproduction in any medium, provided the original author and source are credited. 